\title{
Des écritures individuelles pour construire une représentation collective de la pratique
}

Individual writing aimed at building one collective representation of professional practice.

\section{Vincent Valdelièvre et Pierre Delcambre}

\section{OpenEdition}

\section{Journals}

Édition électronique

URL : http://journals.openedition.org/edc/2383

DOI : $10.4000 /$ edc.2383

ISSN : 2101-0366

\section{Éditeur}

Université Lille-3

\section{Édition imprimée}

Date de publication : 1 juin 1997

Pagination : 135-160

ISBN : 2-0767703-0-8

ISSN : $1270-6841$

\section{Référence électronique}

Vincent Valdelièvre et Pierre Delcambre, « Des écritures individuelles pour construire une

représentation collective de la pratique », Études de communication [En ligne], 20 | 1997, mis en ligne le 30 mai 2011, consulté le 01 mai 2019. URL : http://journals.openedition.org/edc/2383 ; DOI

10.4000/edc.2383

Ce document a été généré automatiquement le 1 mai 2019.

(c) Tous droits réservés 


\section{Des écritures individuelles pour construire une représentation collective de la pratique}

Individual writing aimed at building one collective representation of professional practice.

\section{Entre sens de l'activité et description du quotidien, enjeux et compromis de l'écriture.}

1 Cet article est une analyse d'un dispositif d'aide à l'écriture sur la pratique et l'expérience 1 .

2 Le secteur considéré est celui de l'éducation spécialisée ; un établissement organisé en "groupes» accueille des enfants en grandes difficultés, tant sociales que comportementales. Certains enfants accueillis peuvent être caractérisés comme «border line », " autistes $~_{2}^{2}$. L'établissement, accueillant une soixantaine d'enfants dans des organisations collectives de semi-internat, imagine puis institue à partir de la fin des années 1970, un lieu spécifique pour enfants et adolescents autistes et à structure psychotique (une dizaine d'enfants), un lieu qui se veut dans l'idéologie et les pratiques de l'époque, déterminées comme anti-psychiatriques, et « anti-institutionnelles » : un « lieu de vie ", loin de la vie urbaine mais aussi de la concentration d'enfants de l'établissement, la «maison-mère", qui accueille cinq autres groupes d'enfants, à une quarantaine de kilomètres de là. Il prend comme nom "Groupe rural », est «installé dans une ancienne ferme qui offre suffisamment d'espaces pour que chacun - enfant comme adulte - puisse créer le sien ».

3 Le travail que nous analysons ici est celui de l'aide à l'écriture sur la pratique pour les éducateurs de ce "groupe rural ». Nous rendons compte ici de deux phases du travail, 
celui de la négociation du dispositif d'écriture et celui de la mise à l'écriture. Cette dernière phase s'est close avec la présentation d'un écrit d'une soixantaine de pages sur «la pratique » du groupe rural. Ces écrits ont été collectés et édités, soumis à la lecture d'une vingtaine de lecteurs choisis en fonction de critères définis dans le protocole général de travail.

Le travail que nous avons mené est lié à une double demande. La première est celle de l' équipe ${ }^{3}$, une demande adressée à la direction, consistant à réactualiser le projet « fondateur » de la structure, dans le cadre de tensions que nous expliciterons. La seconde demande émane de la direction ${ }^{4}$ : celle-ci souhaitait que le groupe explicite ce qu'il en était de sa singularité affirmée, et que cette autodéfinition ne se fasse pas dans le champ clos des discussions d'équipe, mais en confrontation avec les discours actuellement tenus sur l'accueil de populations caractérielles et autistes. Le passage par l'écriture lui semblait nécessaire.

5 C'est dans ce contexte qu'un éducateur chef, membre de l'équipe de direction, chargé du suivi de l'activité du groupe rural a estimé qu'une aide à l'écriture devrait s'avérer utile. Il a fait appel à nous (P.D et V.V, membres de Gérico), car nous avions déjà par le passé collaboré avec l'établissement, notamment à l'occasion d'un stage de formation continue sur les écritures obligée des éducateurs spécialisés. Nous avons été, dans cette action d'aide à l'écriture, les instruments d'un compromis.

6 Il faut caractériser avec précision la situation institutionnelle dans laquelle émerge la demande d'écriture : ici l'équipe est dans un mode de fonctionnement ordinaire, elle ne s'estime pas spécialement innovante mais estime avoir besoin de faire le point ${ }^{5}$. L'organisation hiérarchique de l'établissement consiste en une organisation étagée, structurée, avec une direction et un groupe, les deux collectivités étant localisées et identifiées comme imbriquées mais distinctes. Des tensions inhérentes à cette situation pèsent sur les conditions de production de l'écriture sur la pratique.

7 Nous mènerons cet article en trois temps. Tout d'abord nous analyserons un texte qui a accompagné depuis longue date notre réflexion sur les processus d'écriture sur la pratique. Il s'agit d'un texte de G. Jobert, «Ecrite, l'expérience est un capital », publié en 1990 dans la revue Education permanente. Dans les milieux de la formation continue ce texte a eu un certain retentissement, notamment auprès des formateurs qui ont tenté de développer l'aide à l'écriture. Nous aimerions, quelques années après, en faire une relecture et mesurer ainsi l'intérêt des propositions et analyses qu'il contenait.

Dans un deuxième temps nous décrirons rapidement le cadre spécifique dans lequel la démarche d'écriture que nous avons aidée a été mise en place en explorant les négociations et les compromis entre ces trois types d'acteurs : direction, équipe et aide à l'écriture. Nous chercherons à décrire les effets de compromis que ce dispositif a pu avoir sur l'écriture.

9 Nous reviendrons enfin sur les textes produits et leur description de l'activité, en essayant de repérer les difficultés à travailler et écrire la pratique et à verbaliser l'expérience lorsqu'un travail d'écriture collective a lieu sur le lieu de travail, est aidée sur le lieu et dans le temps de travail. 


\section{1. « Ecrite, l'expérience est un capital... ».}

10 Depuis les années 1985, un certain nombre de personnes, regroupés autour d'Education Permanente développaient une série de pratiques d'aide à l'écriture. Récemment encore, une collaboratrice de la revue, chargée de semblables ateliers a fait le point sur ce travail (Dumont 1994). Il s'agissait pour Education Permanente de répondre à des demandes institutionnelles qui leur étaient faites, pour aider des formateurs d'adultes à écrire leur expérience. A l'époque, les formateurs d'adultes étaient en cours de professionnalisation, la Formation Continue ne s'étant développée de manière sensible qu'après 1971.

11 Le dispositif, dont nous ne savons ni s'il était mis en place sur le lieu de travail, ni comment il prenait en compte le temps de travail des écrivants est décrit de la manière suivante :

« ... Des organisations telles que EDF-GDF, l'AFPA ou l'Education Nationale se sont depuis trois ans engagées avec Education Permanente dans un projet qui consiste précisément à faire écrire leurs formateurs à partir de leurs pratiques de terrain. «Faire écrire » signifie en l'occurrence offrir aux formateurs un support spécifique et mettre à leur disposition des personnes capables de les assister dans ce projet d'écriture ». (Jobert, 1990, p. 78).

G. Jobert, dans son texte, insiste sur les enjeux de l'écriture pour le secteur professionnel qui les préoccupaient et les employaient. Son titre, «Ecrite l'expérience est un capital », lui permettait d'unifier trois enjeux de nature différente, trois enjeux concernant des acteurs différents.

13 Le premier enjeu décrit est celui qui pouvait animer les professionnels, les salariés euxmêmes. Pour ceux qui étaient au travail de formation avec des adultes, écrire pouvait comporter des enjeux d'autoformation; la capitalisation est ici celle de l'expérience. Le deuxième était un enjeu pour les employeurs eux-mêmes. Le capital dont il s'agissait était stratégique en ce qu'entreprise pouvait constituer un «capital immatériel ». Enfin pour un autre type d'acteur, la profession, l'écriture pouvait être prise dans des enjeux de constitution et de reconnaissance d'une spécificité professionnelle, de capitalisation des compétences non profanes qu'elle fédère et formalise. Revenons rapidement sur ces trois points et tâchons d'analyser ce que ces dix dernières années nous ont permis d'apprendre à l'égard des enjeux d'écriture pour ces trois types d'acteurs.

\subsection{L'écriture de l'expérience, un enjeu d'autoformation.}

14 L'écriture est inscrite dans un enjeu de formation des salariés eux-mêmes comme personnel en activité. Capitaliser l'expérience, c'était, pour le personnel, éviter de refaire ici les erreurs dépassées ailleurs, mais encore, garder trace de l'expérience pédagogique, dans une situation où les formés peuvent développer des demandes variées, faire valoir les expériences diverses que le formateur ne peut ignorer en « répétant » rituellement des contenus d'enseignement. G. Jobert analyse le «manque à capitaliser » en mettant en avant le fait que les savoirs constitués par la confrontation avec le réel, savoirs issus de la pratique, sont pour partie opaques à ceux-là mêmes qui en sont les détenteurs et les producteurs. Du coup, le dispositif pédagogique met en place une aide à la formalisation et à la capitalisation de l'expérience en deux temps. La première étape consiste en une mise en mots, en quelque sorte faire une séparation de soi ; cette étape doit être aidée, et 
donne, dit-il, un « discours de la pratique, sans guère de profondeur, descriptif, souvent mal ordonné »; ce n'est qu'ensuite, lors d'une deuxième démarche, que l'on va pouvoir produire le «discours sur la pratique» qui replace ce qui précède dans l'espace et le temps, lui donne son contexte, le travaille à l'aide de concepts et de réflexions appuyées sur une communauté de réflexion. Somme toute, si l'écriture permet une capitalisation individuelle, c'est qu'elle interdit le suspens, qu'elle oblige à dérouler complètement sa pensée et, au bout du compte, dans la démarche proposée, transforme l'expérience en connaissance transférable.

Quelle réflexion nous inspire l'analyse de ce premier enjeu ? L'organisation et le protocole d'action pédagogique évoqué par G. Jobert est un dispositif où l'on fait comme si le salarié était un individu qui, lors du dispositif d'écriture, était sorti momentanément de son lieu de travail et se retrouvait dans des groupes, un dispositif typique de la formation continue $^{6}$. Dans le cas évoqué il s'agit vraisemblablement de groupes homogènes de métier, homogènes par l'aspiration à la capitalisation de l'expérience en profession. Or cette dimension, très individuelle, de la capitalisation (la capitalisation par moi de mon expérience à moi), une capitalisation qui s'autoriserait, dans le dispositif Education Permanente, de la volonté collective, partagée, de professionnalisation, nous semble n'être qu'un cas particulier de l'écriture sur l'expérience. En effet, le salarié peut être amené à ne pas écrire sur sa pratique, mais sur la pratique supposée commune, ou mieux, construite comme pratique commune par le travail même de représentation. Cette communauté de référence, celle d'un groupe d'appartenance, pèse sur l'écriture et dans l'écriture peut induire de nombreux flottements dans l'énonciation (Delcambre, 1994). D'autres difficultés à s'approprier comme " personnelle » une pratique existent. Le salarié peut être en effet amené à parler de son travail autrement que comme acte individualisable. Nombre d'activités ont lieu en coordination, dans une équipe. On peut être à deux sur une machine, se succéder dans une prise en charge. La représentation qu'il s'agit de construire dans l'écriture singulière n'est donc pas forcément l'expérience d'une pratique à soi. L'idée que l'écriture est un mouvement de restauration du sujet; l'idée que cette restauration est permise parce que l'écriture permet à chacun de se « réapproprier " (au sens de refaire du propre à soi avec du venu de partout) son activité propre, grâce au travail de formalisation... ne sont pas idées si évidentes dès lors que l'on observe le travail. Quand les dispositifs d'aide à l'écriture s'installent sur le lieu de travail, quand ils sont installés pour les équipes - et non pour des volontaires sortis au comptegoutte, continuité du service oblige! - on est obligé de prendre en compte une dimension collective du travail, la dimension qu'ici nous désignons comme celle d'une équipe.

Cette dimension collective de l'activité est fondamentale car elle s'exerce - souvent simultanément sur deux registres. D'une part, nous l'avons rapidement évoqué à l'instant, le faire n'est pas une activité d'un isolat autonome; mais encore, la représentation de l'activité est, elle aussi, liée à des formes collectives, celles des réunions et des discussions. Nous estimons nécessaire d'insister sur ce point : nombre de représentations de l'activité collective sont préconstruites, que ce soit par les discussions familiales, les rencontres syndicales, les réunions de "métiers »... et par les organisations dont se dotent les entreprises et les services pour réguler, coordonner, évaluer, représenter l'activité. L'équipe est une forme d'organisation du travail et de sa représentation (Delcambre, 1997). Cette remarque vaut pour nombre d'univers de travail. Du coup on se rendra compte que la mise en mots comme séparation de soi, un élément du discours sur 
la pratique qui doit être aidé, dit G. Jobert, a souvent déjà eu lieu. S'il a déjà eu lieu, en quoi doit constituer l'aide? Nous pensons qu'il faut dès lors penser le dispositif d'écriture comme changement de scène énonciative et discursive, comme une manière de rejouer et déjouer certains propos déjà construits. Certes des dispositifs peuvent se donner comme objectif de construire une scène de l'authenticité et de restauration de la parole du sujet, dans une dimension proche de l'analyse, comme on peut le voir dans des dispositifs de formation continue à distance du travail... Mais aujourd'hui toute l'écriture du salarié sur sa pratique ne se fait pas dans de tels cadres.

17 Il nous semble enfin nécessaire de prendre en compte le fait que la capitalisation de l'expérience est un objectif fréquent sur les lieux de travail pour les salariés eux-mêmes. Si l'on prend au sérieux cet objectif même de capitalisation, on sera attentif aux salariés qui, dans des groupes d'expression, des cercles de qualité, des groupes de projet, des équipes innovantes, des équipes semi-autonomes de travail, prennent le risque de passer par l'écriture sur leur lieu de travail. On sera aussi attentif aux collectifs qui veulent prendre l'écriture comme processus de réappropriation collective.

\subsection{L'intérêt des directions pour l'écriture de l'expérience.}

Dans le texte de G. Jobert, le deuxième enjeu est décrit de manière tout à fait curieuse. "Ecrite l'expérience est un capital», tel serait aussi un enjeu pour les entreprises (de formation) elles-mêmes. En effet, les établissements de formation chercheraient quant à eux à construire ainsi un capital immatériel, source de profits. En quoi la réflexion semble-t-elle aujourd'hui étonnante? C'est peut-être que l'on a une autre idée des intérêts économiques des établissements de formation continue et de leurs recherches de « capitalisation ». L'article de G. Jobert prend en compte une réflexion " économique » : le terme de « capital » n'est pas qu'une métaphore. L'acte d'écrire est un investissement (au sens budgétaire), l'écriture est une immobilisation dans un bien précieux : un écrit qui guiderait la pratique et permettrait de ne plus régler au coup par coup les problèmes.

Or, les travaux de P. Moeglin (Moeglin, 1996) et d'E. Fichez (1993) le montrent avec clarté, il y a bien eu, dans le secteur de la formation continue, industrialisation et constitution de capital. Mais constitutions de capital autour des machines à enseigner, autour d'outils de formation : les entreprises de formation ont cherché à substituter le capital au travail.

La remarque de G. Jobert semble ainsi, avec le temps, décalée par rapport aux processus de capitalisation des entreprises de formation. Peut-être faut-il lire son propos comme une adresse aux salariés, aux formateurs : il s'agirait de leur dire, en affirmant que le travail et ses produits appartiennent à l'entreprise employeur (une formule que les enseignants ne supportent pas, estimant qu'ils ont la propriété des productions intellectuelles de leur travail) que ce travail d'écriture intéresse l'employeur qui, en conséquence, ne manquera pas de soutenir l'effort d'écriture. Somme toute l'auteur signalait que la réflexion sur le travail était soutenue par des intérêts communs. Mais il ne traitait pas des problèmes relatifs à la propriété des produits: en fait Education Permanente, par son dispositif, d'offre de formation et d'offre de publication, proposait un espace autre que celui de l'entreprise : les textes qu'elle éditait (les salariés devenant " auteurs ») dans ses suppléments (AFPA, EDF) échappaient pratiquement à la propriété et à la responsabilité éditoriale de l'employeur.

21 Avec l'écriture, nous touchons en effet à des questions qui débordent celle du capital incorporé, celle de l'expérience qui fait le geste sûr, la conception plus rapide, l'analyse 
des problèmes moins angoissée. Une fois écrite, l'expérience est un texte dont les modes de valorisation, de diffusion et de discussion posent des problèmes aux éditeurs et aux auteurs (aux collectifs auteurs aussi). Ce type de questionnement (sur ce qu'est un auteur dans le cadre du travail n'était pas encore abordé dans la fin des années 1980, voir Etudes de communication $\mathrm{n}^{\circ} 16,1995$ et Delcambre 1994). Nous commençons à explorer la variété des cas de figure qui règlent, d'un secteur professionnel à l'autre, d'un métier à l'autre, le droit de signer, de faire circuler un texte produit à son travail, le droit de valoriser sous son nom propre les bénéfices symboliques de la production, de la reproduction et de la mise en circulation d'écrits et leurs retombées financières. G. Jobert a su noter que les entreprises étaient soucieuses de la formalisation de l'expérience par l'écriture. D'autres articles ici même montrent les enjeux actuels pour les entreprises de la formalisation de l'expérience. Il ne s'agit pas seulement d'enjeux de montée en qualification des salariés, mais parfois aussi des enjeux de contrôle (dans le contexte de la qualité), de flexibilité ; il y a aussi des enjeux de présence symbolique dans les champs intellectuels, de notoriété dont les retombées sur le marché de l'entreprise ne sont pas nuls.

\subsection{L'écriture comme capitalisation pour la constitution de professions.}

L'article analysé insiste enfin sur les enjeux de reconnaissance sociale pour les salariés organisés en profession. De fait l'écriture contribue à la définition de l'activité et peut être mise au service des luttes de reconnaissance de professions (Trepos, 1992). Cette analyse est devenue classique : l'écriture permet aux professionnels de rompre le lien de dépendance unique par rapport à l'employeur, en faisant valoir des compétences professionnelles reconnues, voire "sanctionnées" comme qualification. Le capital se constitue donc sur la scène de la profession. Il serait intéressant, pour peu que l'on prenne au sérieux ce dernier enjeu, d'ouvrir la question à d'autres secteurs que celui de la formation continue. En effet, penser le rôle de l'écriture dans la constitution d'une profession avec le seul exemple des formateurs de formation continue risque de ne nous faire penser qu'une étape de la constitution de la profession, celle des premières formalisations des compétences. Si l'on réfléchit au rôle de l'écriture et de l'écrit pour d'autres univers professionnels, on notera que les professionnels peuvent passer à d'autres tâches d'écriture quand les professions sont déjà plus installées, quand les premières écritures de formalisation existent. Ainsi pour les infirmières : le mouvement canadien a permis le développement définitionnel du nursing et des diagnostics infirmiers, manifestant en les formalisant les savoirs, compétences et pratiques spécifiques des infirmières par rapport aux médecins. Trente ou quarante ans plus tard, des générations ont été formées à ces savoirs, utilisent les formalisations disponibles ; d'autres taches adviennent : elles sont d'actualisation; or l'actualisation de l'expérience ne s'écrit plus sous forme de diagnostic infirmier mais par un travail d'écriture dans les revues professionnelles...

Le milieu professionnel est un lieu et un espace désirable pour nombre de salariés : il leur permet d'échapper ainsi à la domination exclusive du lieu de travail, des intentions et orientations de leurs directions, mais aussi parfois de leur collectif de travail, de leur équipe (Pezet, 1993). Mais il faut aussi noter ce qu'il en coûte pour le salarié que d'aller à le rencontre des autres, de la profession. La capitalisation par la profession ne se fait que lorsque le salarié peut assumer ce surinvestissement et s'accomode du déplacement sur 
une autre scène. Le droit à la formation continue peut offrir au salarié une forme moins militante, et moins coûteuse de capitalisation que la participation associative à la construction professionnelle.

Pour le travail ici exposé, la scène du métier est une scène concurrente pour les salariés, et pas forcément désirable. L'organisation en équipe peut rendre la formalisation de la pratique spécifique dans un mouvement identitaire localisé plus désirable que la contribution à la discussion collective dans le cadre du métier. Précisément, dans le cas qui nous préoccupe, on verra que l'équipe peut chercher une fermeture momentanée d'un espace de discussion pour travailler dans l'écriture cohérence et cohésion du groupe, alors que la direction peut vouloir que l'équipe aille à la rencontre d'autres formes de l'expérience et qu'elle se confronte avec d'autres.

Dans les formes d'écriture sur la pratique, la capitalisation de l'expérience peut emprunter la forme de la revendication d'une identité professionnelle, de la réassurance de l'identité professionnelle. L'écriture sur la pratique, pour ceux qui ont vécu la socialisation secondaire (Dubar, 1991) dont la formation professionnelle a aidé à la construction, peut aider à poursuivre, sur le lieu même de la pratique de l'équipe, la référence à une pratique de métier. Référence aux textes fondateurs, aux noms des théoriciens de référence, usage des concepts professionnels, éléments thématiques, nombre d'éléments discursifs reconstruisent sur le lieu de travail une telle référence au métier. Les enjeux de capitalisation sous la forme "professionnelle » jouent donc non seulement comme recherche de "reconnaissance sociale», pour reprendre l'expression de G. Jobert, mais comme recherche de reconnaissance locale d'une dimension socialeprofessionnelle (et en tant que telle séparée de l'expérience profane, captée comme spécialisée par un groupe) de l'activité.

\section{L'écriture sur la pratique dans le cadre du travail comme compromis.}

\subsection{Le passage à l'écriture pour dire l'activité, une double demande.}

Notre intervention d'aide à l'écriture est liée à l'histoire d'une double demande et à l'installation d'un compromis que nous avons contribué à formaliser. En effet, nous avons été contactés par le chef de service, travaillant ordinairement dans les locaux de la direction et travaillant deux fois par semaine dans l'équipe du groupe rural. Pour celui-ci, à sa place, une intervention de "spécialistes " connus de l'écriture, qui étaient déjà intervenus dans un cadre de formation continue, pouvait aider à stabiliser un compromis qui donnerait lieu à une production et permettrait d'avancer la réflexion sur la pratique du Groupe Rural.

* Le groupe rural et ses éducateurs, plus de dix ans après son installation, désirait quant à lui refaire le point sur son projet. Le contexte de cette volonté collective était un conflit latent sur le processus d'admission des enfants pris en charge. C'est en effet la direction de l'établissement, assistée de ses psychiatres, qui procède aux entretiens d'admission suite aux demandes venant des commissions départementales compétentes. Le groupe déplorait le manque de maîtrise qu'il avait de ce processus et le fait qu'en conséquence on lui demandait de prendre en charge des enfants dont l'accueil, de son point de vue, ne lui permettait plus de travailler en cohérence avec le projet fondateur. Le chef de service 
était central dans le jeu de responsabilité concernant ce glissement d'activité, comme représentant de la direction. Le groupe rural s'appuyait sur un projet établi à sa fondation, légitimé dans un conseil d'administration pour faire valoir l'autonomie de décision, ou le contrôle sur les admissions qu'il réclamait. On note donc une volonté de reprise en main du « matériau de travail » pour une réaffirmation du « sens du travail » spécifique que l'ancien projet définissait pour ce « lieu de vie ».

Mais fallait-il encore que l'ensemble de l'équipe soit une force mobilisable sur une base collective. Or depuis dix ans, le personnel avait quelque peu "tourné», et à côté de certains fondateurs encore en place, des nouveaux venus cherchaient à développer leur activité professionnelle, s'appuyant tantôt sur une interprétation du projet différente de celle des fondateurs, tantôt sur une conception actualisée de la prise en charge de ce type d'enfants que leur formation et leur qualification professionnelle leur permettait de soutenir. L'ensemble des personnels du groupe s'accordait sur la nécessité de «faire le point» sur l'activité commune et sur le sens que chacun donnait à ses pratiques ordinaires ${ }^{7}$. L'écriture était donc l'occasion pour l'équipe de poser chacun, de manière individualisée, sa parole et d'en discuter. La question de l'identité du groupe se posait tant sur le versant, ordinaire, répétons-le, du sens des actions, que sur le versant collectif de l'orientation de l'action et de sa maitrise par le contrôle des admissions.

* La direction, de son côté, ne gère pas seulement un groupe, mais six. Dans le cadre des pratiques actuelles de direction, dans le secteur sanitaire et social, la nomination d'un nouveau directeur, s'accompagne d'un projet, d'un discours d'orientation. Installée depuis plusieurs années, la direction avait réorganisé les cinq groupes de la maison mère, laissant le groupe rural poursuivre son activité. Mais les conflits sur les admissions avaient réactualisé la question de l'articulation entre la pratique autonomisée de ce collectif et la politique affirmée dans le projet du directeur. Pour la direction la volonté d'autonomie définitionnelle du groupe rural lui paraissait trop importante: elle souhaitait que cette équipe pense son activité en rapport avec le contexte politique qui pesait sur l'ensemble de l'établissement et que le groupe rural se définisse en tenant compte des enjeux globaux de la prise en charge d'enfants autistes, enjeux concernant tant les flux d'enfants accueillis, que sur les pratiques éducatives à l'égard de ces enfants. Or ces politiques se définissent non dans le cadre des groupes et des établissements, mais bien dans le cadre d'une politique d'action sanitaire et sociale mouvante, échappant au contrôle de chaque établissement en particulier.

Par l'écriture, la direction souhaitait que la démarche de redéfinition du projet se développe et quitte la sphère des échanges internes au groupe. En effet l'écriture exigerait une formalisation des propos leur permettant d'échapper aux humeurs des discussions lors de réunions. Mise à l'ordre du jour, la description des pratiques pourrait devenir un objet de discussion critique permettant de saisir ce que cette direction sentait comme "insaississable singularité autoproclamée du collectif» (la formule est de moi, P.D). L'écriture permettrait enfin de confronter les avis de l'équipe avec d'autres discours contemporains, que ce soient ceux d'autres équipes, ailleurs, ou ceux d'autres acteurs partenaires de la définition de l'action sociale en direction de tels enfants. La direction pouvait donc vouloir que l'équipe rentre dans un champ, sinon économiquement concurrentiel, du moins symboliquement pluriel, de définition de l'action. 


\subsection{Un dispositif d'écriture comme compromis.}

31 A l'issue d'une phase de discussion et de négociation qui a duré six mois, notre intervention a été stabilisée sous la forme d'un contrat. Ce dernier définissait les objectifs généraux de la manière suivante :

1. Le groupe rural de $\mathrm{C}$. a souhaité travailler en équipe pour « faire le point sur sa pratique ». Un passage par l'écriture permettrait aux différents salariés d'expliciter le mode et les principes de fonctionnement du travail du Groupe rural.

2. Par ce travail collectif soutenu par la direction de $l^{* * *}$, l'équipe vise à mieux situer la pratique collective actuelle par rapport aux politiques d'accueil d'établissements similaires.

3. Enfin il s'agit de clarifier les enjeux professionnels caractérisant l'évolution actuelle de la prise en charge des populations concernées pour aider les salariés du groupe rural à participer à la discussion sur la politique de leur établissement.

Trois phases étaient prévues: la première de mise au point et de négociation concernant l'action; la seconde d'écriture individuelle et collective, de discussion en équipe restreinte et élargie; la dernière de travail sur les pratiques et enjeux actuels de la prise en charge de la population concernée. Ce sont les deux premières phases qui sont analysées dans cet article. La phase d'écriture devait se clore par une lecture des écrits réalisés dans un cadre professionnel, lors d'une demi journée de réflexion accueillie dans une école d'éducateurs de la région ; trois types de lecteurs furent sollicités : des collègues de l'établissement, des professionels du secteur, des chercheurs de GERICO ; cette journée a effectivement eu lieu en septembre 1996.

Nous avons essayé dans le dispositif de régler les problèmes des formes de l'échange dans le groupe, de destinataire. Nous avons recherché une production immédiate, les échanges sur les textes étant eux étalés dans le temps (les textes étant l'objet des échanges d'une serie de réunions, au rythme de deux textes par réunion). Ces réunions avaient lieu sur le temps de travail, dans les locaux mêmes du groupe, un éducateur restant avec les enfants durant ce temps. La règle d'échange voulait valoriser la dimension critique de l'échange : les remarques devaient amener l'auteur du texte à clarifier ou approfondir ce qui paraissait obscur ou non abouti aux autres membres du groupe. L'auteur était soumis quant à lui à une règle d'écoute silencieuse, pour éviter que les réunions soient l'occasion d'un jeu de remarques critiques suivies de réponses défensives. Les règles du travail ont été explicités lors des réunions préalables et reformulées au cours du travail, ainsi :

L'expression recherchée est celle de l'équipe, sans toutefois imposer au consensus universel: chaque expression garde la marque de son auteur, en cas de besoin plusieurs expressions sont nécessaires pour rendre compte d'une diversité dans l'action ou dans les points de vue. [...] Le groupe échange sur la base de premiers textes déjà écrits [...] Les questions se font sur trois axes : réactions personnelles, recherche de faits et informations qui peuvent servir à approfondir ou élargir ce qui est énoncé, on se demande ce qu'il faudrait connaître de ce que d'autres ont fait dans d'autres établissements pour comparer [...]. L'écriture est orientée : chacun aura écrit à l'intention d'un ou de plusieurs destinataires, que la lecture puisse se faire en connaissance de cause dans le groupe [...] Les thèmes d'entrée (une liste a été établie lors d'une première réunion) donnent des idées d'écriture. Une autre approche est possible par des analyseurs de la vie quotidienne (ex: l'enfant et l'agenda ; bonjour et au revoir : ce qui se passe quand on arrive au travail ou quand on le quitte; les portes, escaliers, couloirs...) V. Valdelièvre, compte-rendu de réunion. 
Un dispositif qui laisse néanmoins chaque écrivant devant ses choix et ses incertitudes

Personnellement, j'ai l'impression d'avoir loupé des étapes: n'y avait-il pas nécessité d'avoir dans un premier temps des échanges entre nous autour de nos pratiques? Qu'est-ce qui fait nos différences et quels sont les points d'achoppement. A qui s'adresse-t-on? Certes tous ces points ont été abordés, mais rien ne semble avoir été arrêté. Chacun se déterminera devant sa page blanche. Comme je l'ai dit lors de la réunion du xx, je préfère avoir un cadre pour écrire. Pour parler de mon travail à $C$., je présenterai donc l'atelier que j'anime le vendredi après-midi. B. (éducatrice).

\subsection{Description du quotidien et sens de l'activité.}

Si l'on adopte le point de vue de l'équipe, des écrivants, à l'intérieur du cadre rapidement évoqué, il nous semble que l'écriture elle-même est effectuée sous le signe du mixte, de la tension entre deux formes d'écriture : description du quotidien et sens de l'activité. Indiquons quelques enjeux.

Le cadre global voyait en présence deux approches. L'une visait à réaffirmer les principes identitaires, à refonder l'utopie du projet, la singularité du type de prise en charge par l'équipe, la spécificité de la philosophie commune et des principes d'action. L'autre approche, c'est nous-mêmes qui l'avions largement développée, en ce qu'elle nous semblait un espace de travail «de compromis » : nous avons valorisé une approche qui consistait à décrire le quotidien, les pratiques ordinaires de travail. Une telle préconisation nous semblait pouvoir ouvrir à une autodescription qui ne soit pas toute entière dominée par l'autoaffirmation identitaire défensive; elle nous semblait aussi pouvoir ouvrir à l'échange en ce que chacun aurait par une description assez «plate » donné à voir une expérience de travail ordinairement non accessible aux autres. Dès lors si, au bout de l'échange, les uns et les autres arrivaient à reconnaître du « sien » dans « les autres ", peut-être un collectif pouvait-il s'y retrouver, voire y trouver quelques éléments de sa singularité collective.

Néanmoins, en opposant comme nous venons de le faire l'intériorité d'un projet de groupe et l'extériorité de notre intervention, on rend mal compte de ce qui a fait accepter notre dispositif. Le compromis est lié à des ambivalences dans les choix à faire et le désir de représenter la pratique. Une différence travaillait le groupe dans le choix du mode de description/définition de la pratique. Pour les salariés eux-mêmes décrire la pratique quotidienne c'est se donner l'espace d'explicitation de pratiques qui ne sont pas forcément connues (un espace d'interconnaissance donc), c'est aussi avoir l'occasion de pouvoir dire ce qu'on fait sans nécessairement maîtriser les concepts, les références, l'arsenal idéologique et professionnel de l'orientation affirmée de l'action. Dire la pratique en décrivant le quotidien, c'était encore une position "épistémologique " partageable, pour laquelle la pratique n'est pas seulement orientation, choix et définition, mais aussi manque à dire, expérience qui advient, éléments donnés à vivre. Dès lors, prendre le temps de raconter ce qui advient pour en verbaliser une expérience est un élément fondateur de la réappropriation de l'activité, de la reconnaissance du travail comme réinterprétation des faire, comme adaptation perpétuelle aux événements. Et, encore une fois, avec les enfants qui vivent dans l'espace institué comme cadre, avec ces relations où le sens échappe plus souvent qu'il ne s'ordonne, une telle méthodologie de la description, ordinaire, pouvait être aussi une "entrée dans l'écriture de la pratique ». Ainsi, dans un moment exploratoire et relativement pacifié, certains membres de l'équipe 
ont pu tenter de dire ce qui advient, mettant un moment en suspens la construction identitaire et la refondation du projet.

Reste que cette méthodologie est elle même une orientation et une utopie : elle suppose autre chose que le débat d'idée, l'échange hic et nunc sur les rationalités présentes et reconnaissables. Pour que cette utopie trouve un moyen de faire progresser le travail du dire sur la pratique, il fallait encore que le jeu des échanges, des questions sur le travail de l'autre trouve son modèle communicationnel. Le bilan de cette pratique reste à faire. Nous nous contenterons dans le cadre de cet article de revenir sur les textes produits, en prenant comme objet de réflexion les textes finalement édités, mis en circulation au termes des échanges. Que disent-ils de la pratique?

\section{Le discours des pratiques: quelques caractéristiques des écrits réalisés.}

On peut reprendre l'approche de D. Hameline (1979) à propos de la description, dans laquelle il propose cinq degrés ou niveaux d'approche des situations, à l'intérieur desquelles la description se situera dans une fourchette. Reprenons l'exemple.

Dans une classe de maternelle, l'institutrice s'approche d'un enfant, l'observe pendant quelques instants et peut dire :

1. - « je vois que la sécrétion de liquide qui balaye tes globes oculaires augmente. Tes angles larmiers sont engorgés. Le liquide déborde la paupière inférieure et s'écoule sur tes joues. L'extrémité de ton nez effectue, par soubresauts, des plissements qui dégagent l'ouverture des narines d'où émane un bruit d'aspiration..."

2. - « je vois que tu pleures"

3. - « je vois que tu veux me dire quelque chose»

4. - « je vois que tu as du chagrin »

5. - « je vois que tu piques encore ta crise»

Cinq attitudes caractéristiques d'observation et de description que l'on peut résumer ainsi : le relevé des indices élémentaires, la synthèse de ces indices, la projection d'une intention, l'interprétation des sentiments, le jugement de valeur.

41 Si l'observation est toujours la même (tous les indices énumérés), le traitement de ces indices prend des formes bien différentes: en l'associant à d'autres opérations spécifiques, le praticien se distingue du chercheur. Son objet n'est pas d'abord de faire la preuve que ce qu'il avance est vrai (ou vérifiable ou falsifiable) mais d'énoncer quelque chose qui fonctionne, c'est à dire quelque chose d'apte à produire des effets dans la relation. Le praticien est « un observateur de comportement [qui] demeure une machine à fabriquer du sens. »

Notre a priori de lecture était que l'on devait trouver dans les écrits, en termes de description de pratiques éducatives, une transposition de la situation pratique dans laquelle sont les adultes et les enfants : en interaction. Et donc, des énoncés dans lesquels on trouverait l'adulte et les enfants en action, des énoncés articulés entre eux dans une logique d'action, tous les autres énoncés venant compléter ces énoncés de base, en termes de réflexions, d'analyses, de commentaires, etc.

On peut schématiser ainsi ce qu'on trouve: des informations sur les enfants, sous forme d'informations objectivées (Laurent est furieux ou C. vient de rentrer). Cela est redoublé par des énoncés qui parlent des enfants, dans lesquels le sujet de l'action est abstrait ou en 
retrait (La voix d'O. se fait entendre ou Une cabane est construite avec l'aide d'un adulte). Tout ceci constitue les deux tiers du discours.

On trouve ensuite des informations sur les éducateurs, en moins grand nombre

Le jour est à peine levé. L'éducateur qui est resté cette nuit est dans la cuisine,

préparant l'accueil du petit déjeuner, moment essentiel de la journée...

Reste une poussière d'énoncés divers, parfaitement impersonnels (il ne s'agit pas de se substituer à l'école ou Il existe bien des mots dans la lumière de cette matinée) ou d'une très grande généralité

pour établir cette relation, l'être humain doit avoir conscience de ce qui se passe,

pouvoir exprimer ses besoins et les partager avec autrui

ainsi que quelques citations d'enfants (Est-ce que tu vas m'obliger? ou t'as pas à me commander...).

Il est remarquable que seuls deux énoncés prennent pour sujet nous à savoir les enfants et l'éducateur (Nous sommes passés à la présentation des logiciels et nous avions [étions ?] convenu de poursuivre cette activité). Ils sont tous deux tirés d'un texte sur un atelier. Les autres nous désignent régulièrement les éducateurs.

Le discours des pratiques porte sur la pratique, c'est à dire sur ce que l'on fait quand on exerce son métier. Ce métier est un métier de la relation, y sont à l'œuvre au moins deux acteurs : l'éducateur et l'enfant. On s'attend donc à ce que le discours fasse une part importante à ces deux sujets. Ce qui est le cas. Voyons selon quelles modalités.

49 a) La place de l'observation. Les enfants sont observés dans leurs gestes, attitudes et comportements, ceci nous est rapporté soit directement (S. passait beaucoup de temps autour de l'écriture), soit indirectement

Les aller-retours en voiture sont l'objet d'importantes convoitises. Qui sera devant?

Qui aura la place du milieu ? Cela amènera des alliances ou des exclusions [...]

Ce type d'énoncé est le plus fréquent, plus d'un tiers du total.

51 b) Le rôle de l'adulte. Les éducateurs sont protagonistes de l'action (J'ai pris l'initiative de me rendre, un après-midi par semaine, au site informatique de T.) Cependant ils sont souvent absents des énoncés qui leur reviennent

Prétendre ne les aborder [les apprentissages] qu'après une solution des problèmes psychologiques est un non-sens, puisque ce serait nier les liens [...] ou Parfois on se surprend, bien involontairement [...] à être les réceptacles des recherches de chaque enfant.

Par ailleurs, rares sont les interactions. On en trouve quelques unes mais très rares

Quand je suis avec lui, il répond aux questions que je formule à partir des jeux. ou Après quelques séances, $\mathrm{S}$. refusa d'y participer, ses seuls arguments se résumant à «J'ai pas envie ». La raison exprimée ne me suffisant pas, j'insistais sur la notion de contrat que nous avions passé ensemble.

Ces deux exemples proviennent d'un texte sur le travail en ateliers.

c) La recherche du sens. Au delà des protagonistes de l'action, au delà du compte-rendu de l'action, une part très importante du discours des pratiques est orientée sur la recherche du sens de ce qui est observé (peut-être pour se rassurer sur notre propre utilité ou il reste dans la pièce, semblant observer ce que font les autres). Cette dimension est parfois explicite, comme ci-dessus, le plus souvent cette recherche est noyée dans la description ou prend la forme de réflexions qui ne cessent de s'entremêler, par exemple, au discours descriptif donné sur le mode du récit (le visage fermé, prenant les traits d'un enfant qui aurait 
grandi trop vite ou le visage de G. vient de s'épanouir). Ce sont les énoncés de ce type qui sont le plus fortement articulés au reste du discours (comme si, comme pour, peut-être parce que, etc.).

d) Le temps du discours, principalement au présent et, en complément, au passé, rarement au futur, est finalement décontextualisé. A l'exception de certaines actions au passé, relativement précises, l'ensemble des énoncés ne se situe guère dans le temps, plutôt dans la permanence: on a le plus souvent un discours d'état qu'un discours d'action, des propositions juxtaposées.

e) Par ailleurs, une interrogation importante subsiste sur le statut à donner à toute la partie du discours constituée d'énoncés qui font abstraction du sujet de l'action (Une maison qui accueille des enfants ou Comment une atmosphère [...] s'est-elle transformée ?) On a là plusieurs procédés discursifs, dont le plus fréquent est celui qui donne vie et existence quasi autonome à des éléments inanimés ou immatériels (la maison, l'atmosphère, mais aussi la décision, la peur, l'envie de grandir, etc.). On peut analyser cette abstraction comme un retrait des acteurs dans la représentation de leur action; mais il peut aussi s'agir d'un passage à l'analyse, dont la nécessité est pour nous une évidence mais dont le poids relatif est une découverte.

f) Autre attente déçue, les actions, les faits et gestes des professionnels dans les situations professionnelles. Pour avoir pendant plusieurs années fait travailler des formateurs d'adultes sur l'analyse de pratiques dans un dispositif qui les contraignait à faire des relevés d'informations sur ce que font les protagonistes dans l'action, démarche fondée sur le postulat qu'il y a intérêt à identifier et à analyser les actions et décisions des acteurs en situation, à la lumière de la notion de pratiques sensibles, nous souhaitions amener les éducateurs du groupe rural à ce type de travail. Peine perdue pourrait-on dire en première approche. Je est modérément présent dans le discours, qui plus est, il est plus souvent sujet de réflexions et d'interrogations que sujet d'une action ou interaction. Inversement, les actions sont souvent détachées de leurs acteurs et deviennent donc sujets d'un discours dans lequel l'action n'importe pas plus que le sens que l'on peut y trouver. Nous est également présent, également à petite dose, et également sujet de prédicats qui ne désignent pas l'action par ce qu'elle est (ce qu'elle a été) mais par ses intentions ou ses résultats attendus ou supposés (parce que nous lui avons offert d'autres images possibles ou nous avons essayé de renouer des relations filiales). Ces énoncés peuvent très bien désigner quelque chose de réel et de vérifiable, ils ne contiennent aucun élément propre à les infirmer ou valider. Ils ne peuvent donc guère présenter d'utilité directe dans une analyse des pratiques. Ils nous mettent cependant sur la piste d'une articulation des pratiques individuelles au sein d'une pratique collective. Mais de quoi s'agit-il, comment cela se fait-il ?

58 g) Par l'utilisation de la forme passive (Une maison est construite avec l'aide d'un adulte) ou pronominale ( $L a$ voix d'O. se fait entendre), on obtient un effet assez marqué de déréalisation des actions au profit de faits ou de signes matériels. Cette construction n'est pas exceptionnelle, elle revient régulièrement dans les textes, comme pour marquer que les actes ne sont peut-être pas plus significatifs que les traces qu'ils laissent.

En résumé, si l'on reprend les catégories d'Hameline signalées en début de cette partie, nous avons ici un discours des pratiques qui gomme volontiers les acteurs, qui parle peu de ce que fait l'éducateur individuel (il en parle de façon parfois précise, souvent allusive ou globalisante), débouchant sur une énonciation à nouveau des intentions et sur la recherche du sens, sur un travail de compréhension de ce que signifient effectivement les 
gestes, silences, paroles, attitudes et comportements des enfants. Les enfants peuvent être décrits au passé, sur le mode du récit, ou au présent, comme pour insister sur la permanence qui les caractérise ( $L$. vous a dit bonjour ou on parle de psychose). Quelques notations au futur désignent soit ce qui attend tel enfant (C. devra essayer de continuer à se construire), soit se rapportent aux intentions du collectif (Le groupe rural pourra être un espace transitionnel où l'enfant accueilli pourra se poser), manière éventuellement personnelle et prudente d'exprimer des intentions, des éventualités alors que l'équipe ne les a pas encore validées. Enfin on a repéré beaucoup d'interprétations, pas ou peu de jugements de valeur.

\section{Le discours de la pratique : les difficultés à l'œuvre.}

En quoi les productions des éducateurs du Groupe Rural donnent-elles des éléments pour une analyse de pratiques?

61 Tout se passe comme si le caractère exceptionnel des interactions était représentatif du mode de fonctionnement de la relation dans ce lieu de vie entre adultes et enfants. L'éducateur ne doit pas tant réagir à ce qui se passe que le noter, l'enregistrer, relever les indices complémentaires pour le comprendre, et ensuite, dans un autre domaine de la pratique (les réunions, évoquées mais non décrites dans ces textes), en faire état et le partager pour en construire le diagnostic qui permettra de poser de nouvelles lignes de conduite. Du point de vue de l'action et du temps, adultes et enfants ne vivent pas ensemble. Des êtres se croisent, ils s'observent, essaient de se comprendre et chacun conserve beaucoup de choses pour soi.

On peut estimer qu'il en est de même pour les enfants. Pour tout ce qui est prise en charge des impératifs de la vie quotidienne, les éducateurs font à manger, font la vaisselle, le ménage devant les enfants qui enregistrent le fait mais, s'ils réagissent, s'abstiennent souvent de s'impliquer dans l'action, d'y prendre part, gardant pour eux ce qu'ils pensent. Schéma inutilement ironique ou partiel, dans la mesure où les contributions sur les ateliers montrent que là, précisément, c'est d'interactions qu'il s'agit. Non plus des êtres qui se croisent mais des acteurs à l'œuvre.

\section{A quoi tiennent les difficultés de l'écriture?}

63 a) Les enfants ne peuvent pas être sujets: c'est leur souffrance qui est l'objet des pratiques éducatives, cette souffrance s'objective dans des gestes, des paroles, des silences, des attitudes, des comportements dont on ne pourra faire quelque chose que lorsqu'on y aura mis des mots, mis du sens. De ce point de vue, donc, ils ne sont guère sujets de l'action et il est aussi juste de dire qu'une cabane a été construite avec l'aide d'un adulte que de dire que $\mathrm{L}$. a construit une cabane avec l'aide... Inversement,

lorsqu'il n'est plus prisonnier de son univers d'objets, L. peut s'adresser à nous et son langage devient beaucoup plus clair, plus compréhensible, plus ancré dans une dimension humaine...

Lorsqu'il va mieux, cet enfant redevient sujet de son discours et de celui de l'autre.

b) L'éducateur individuel ne doit pas être sujet: la production du sens est un processus d'analyse avec les psys, l'éducateur individuel y contribue. Il apporte les observations qu'il a faites dans son temps de travail, en accompagnant des enfants. Il apporte 
également ses hypothèses et ses interrogations. Il est un membre actif de ce travail de recherche de sens mais ce travail est celui du collectif (l'équipe). A titre personnel et avant la réunion, son discours sera émaillé d'interrogations, de formules de prudence ( comme pour nous montrer qu'il cherche des racines ou qu'il a grandi). L'éducateur est là pour accompagner l'enfant, il n'a pas mandat pour une action thérapeutique. Il est bien plus légitime, de ce fait, d'être le sujet de l'observation que celui de l'action.

c) Ce ne sont pas les actes ou les paroles qui comptent mais l'interprétation qu'on pourra leur donner. Nous avons déjà établi ce point. Seulement, vu que l'interprétation légitime est celle de l'équipe, la machine à produire du sens, ne peut pas fonctionner correctement, faute de légitimité. Ainsi trouve-t-on tous les énoncés incertains qui donnent au discours son étrange tonalité d'affirmation dans le doute, une déréalisation des faits rapportés, le caractère vague et décontextualisé des descriptions

Le nœud de sa problématique semble s'articuler autour de la question de sa venue au monde, avec la peur de perdre sa mère et la question de la présence-absence de son père.

67 On peut se demander si le dispositif d'écriture, obligeant de poser individuellement l'observable comme représentation construite n'est pas, pour les éducateurs, un dispositif qui transgresse les règles ordinaires du travail collectif d'analyse et laisse les éducateurs démunis.

d) Il y a peu, voire même il ne doit guère y avoir d'interactions. Le projet du groupe rural mentionne que ce lieu est un lieu de vie, non un lieu dédié à des activités thérapeutiques. Les éducateurs ont pour mission d'accompagner les enfants dans le quotidien dont les contraintes, simples et évidentes, stables, seraient liées aux rythmes et non à l'arbitraire. Ces contraintes doivent fournir un cadre compréhensible dans lequel les enfants tenteront de recomposer leur personnalité gravement perturbée (c'est moi V.V. qui reformule). Dans le respect d'un tel contrat, l'éducateur s'efface derrière l'observateur et nous relate un moment de la vie au Groupe Rural de la façon suivante :

Parfois, comme si les souffrances ou les manques liées à son histoire familiale faisaient irruption, il se met à insulter la femme présente ce soir-là. Ou bien il se met à contester cette autorité qu'il reconnaît et qu'il dénigre en s'adressant à l'homme: "C'est moi le plus fort, t'as pas à me commander... Je fais ce que je veux... » Alors il s'en va, claquant la porte, comme pour tenter d'échapper aux autres et à lui-même, perdu dans une fausse liberté...

Et puis il revient, visage fermé ou prenant les traits d'un enfant qui aurait grandi trop vite, avec cet appel à peine déguisé : Viens t'occuper de moi !

69 Pas plus dans ce passage que dans ce qui précède ou ce qui suit, l'éducateur ne se manifeste comme ayant une action quelconque en regard de ce que fait $\mathrm{C}$.. La mention des éducateurs, de leur action, de la pratique professionnelle à l'œuvre est: Pourtant nous avons essayé de renouer, de restaurer des relations filiales avec ses parents.

70 Cependant, lorsqu'il s'agit d'atelier, le discours change : l'enfant est sujet, l'adulte se montre en action, des interactions se produisent. Reste très présente la grande incertitude sur le sens qu'il faut donner à ce que font les enfants, et cette incertitude est clairement et précisément celle de l'auteur du texte (moins de nous, moins de on, moins de comme si ; c'est je qui pose des questions). Or, l'un des axes de discussion au sein du collectif des éducateurs portait régulièrement sur la mise en suspicion des ateliers, comme pratique volontariste ayant tendance à outrepasser ou renier la vocation du groupe rural. Les ateliers, lieu à partir duquel se formule plus fermement un discours des pratiques, sont un lieu limite de la pratique: ce n'est pas si conforme que cela à la 
philosophie du lieu de vie, c'est faire le pari de l'intervention éducative contre celui de l'accompagnement. Le lieu de l'action et de la certitude pratique est également celui où le praticien est isolé et renvoyé à lui-même, dans l'incertitude sur la pertinence de son action eu égard aux orientations du groupe rural.

Il n'en reste pas moins, selon nous, que si une part essentielle de la pratique professionnelle se développe au cours des réunions, cet objet doit absolument être décrit et analysé par les éducateurs, s'ils veulent continuer de faire le point sur leur pratique, comme ils l'ont annoncé eux-mêmes, s'ils veulent rendre leur pratique comparable à ce qui se fait ailleurs, comme y incite la direction.

On peut anticiper sur une difficulté importante: quelle est la place de chacun dans la réunion, et dans quelle mesure chacun des participants peut-il parler avec pertinence de ce qui s'y passe ? Décrire des réunions, c'est peut-être risqué, y a-t-il moyen de prendre des garanties pour y parvenir et rendre analysables ces situations décisives de la pratique professionnelle? A ce sujet, nous avons formulé des hypothèses et des propositions pour une observation dans d'autres lieux professionnels, en deuxième phase de l'opération, et pour la mise en œuvre d'observations méthodiques et cumulables: de l'observation «armée »en tant qu'aboutissement et prolongement logique d'une première phase d'observation intuitive.

\section{BIBLIOGRAPHIE}

Education Permanente, (1990), n 102, Les adultes et l'écriture.

Etudes de Communication $\mathbf{n}^{\circ}$ 16, (1995), Pratiques d'écriture et champs professionnels (3) Petites fabriques d'auteur.

Delcambre, P., (1994), Ecrire sur sa pratique en milieu de travail : à la recheche d'un espace de discussion, in Education Permanente, $n^{\circ} 120$, « Ecriture, travail, formation », p. 73-94.

Delcambre, P., (1997), (à paraître), Ecriture et communications de travail, Presses Universitaires du Septentrion.

Dubar, Cl., (1991), La socialisation. Construction des identités sociales et professionnelles, A. Colin.

Dumont, M., (1994), L'aide à l'écriture, un processus aléatoire, in Education Permanente, $\mathrm{n}^{\circ} 120$, « Ecriture, travail, formation », p. 95-106.

Fichez, E., dir, (1993), Etudes de Communication n 14 « Education, formation, le temps de l'industrialisation?».

Hameline, D., (1979), Les objectifs pédagogiques, ESF.

Jobert, G., (1990), Ecrite, l'expérience est un capital, in Education permanente n 102, avril 1990, p. 77-82.

Moeglin, P., (1996), Polyvalence du facteur technique dans l'industrialisation de la formation, Actes du 10ème congrès national des Sciences de l'Information et de la Communication, p. 417-432. 
Pezet, V., Villatte, R., Logeay, P., (1993), De l'usure à l'identité professionnelle, Le burn-out des travailleurs sociaux, Ed. TSA.

Revuz, Ch., (1990), Moi, écrire ?... Je..., in Education permanente $\mathrm{n}^{\circ}$ 102, avril 1990, p. 83-100.

Trepos, J.-Y., (1992), Sociologie de la compétence professionnelle, Presses Universitaires de Nancy.

\section{NOTES}

1. Cet article est issu d'une action d'aide à l'écriture menée en collaboration par V. Valdelièvre et

P. Delcambre. Les deux premières parties de ce texte ont été rédigées par P. Delcambre, la troisième par V. Valdelièvre. Le nous utilisé renvoie à la responsabilité conjointe que nous assumons pour l'ensemble des propos tenus.

2. Notre description est celle liée aux catégories que la profession utilise. Au cours de cet article, nous aurons l'occasion de repérer combien, une fois la désignation opérée, à des fins d'orientation, de construction des modes de prise en charge, dans le cadre d'un marché des établissements d'accueil, la question qui consiste à savoir ce qu'est «en pratique » un enfant accueilli reste à discuter.

3. Dans le secteur on appelle équipe tantôt le collectif d'éducateurs (on dira ici le groupe d'éducateur), tantôt une forme d'organisation du travail où interviennent aussi la hiérarchie, et des «psys». Ici le dispositif d'écriture consacre comme unité le groupe d'éducateurs qui travaillera dans l'écriture indépendamment des psys qui interviennent pourtant régulièrement dans les locaux du groupe rural.

4. Dans le secteur, les directions sont issues de la profession d'éducateur elle-même. Elles sont liées à un "management de proximité ", même si, aujourd'hui elles peuvent être soumises à des injonctions à développer une pratique de gestion financière et comptable plus proche des nécessités de gestion administrative. Les directions restent garantes de la politique d'accueil et du projet d'établissement et, comme telles, sont en discussion permanente avec les conseils d'administration des associations gestionnaires ainsi qu'avec les niveaux départementaux de définition des politiques d'action sociale (inspecteurs DDASS).

5. La situation décrite est donc sensiblement différente de celle étudiée ici même par P. Delcambre dans l'article Compte-rendu d'activité et écriture collective de l'expérience, où l'équipe est un collectif ayant son unité d'action et ses marges de manoeuvre par rapport à une direction générale gérant un grand nombre d'établissements dans une politique coordonnée.

6. Dans ce numéro d'Etudes de Communication, on retrouvera dans l'article de J. Donato un écho de ces dispositifs mis en oeuvre à distance du travail, dans des groupes fédérés par l'activité de «formation continue ».

7. L'accueil permanent d'enfants autistes pose avec une force considérable la question du sens de l'activité. Quand des enfants grognent, ont une activité dont ils ne peuvent verbaliser le sens, quand des personnes prises en charge collectivement interrogent à chaque instant la valeur de l'action éducative en ce que précisément aucune coopération ne permet de donner du sens, on imagine combien la description de l'activité et l'affirmation du sens de l'activité sont à la fois centrales et ordinairement problématiques. 


\section{RÉSUMÉS}

Dans cet article les deux auteurs analysent un dispositif d'aide à l'écriture sur la pratique mis en place dans un établissement de l'enfance inadaptée, dans le temps de travail. Après une partie générale où ils tentent de décrire les enjeux actuels qui amènent des salariés et leurs directions à rechercher le passage par l'écriture pour la formalisation et la capitalisation de l'expérience, ils explicitent les conditions locales dans lesquelles direction et collectif d'éducateurs ont trouvé un compromis. Enfin ils analysent les difficultés de cette écriture : certaines proviennent de la verbalisation même d'un travail d'éducation de jeunes "autistes». D'autre part, l'écriture, comme dispositif, a autant d'effets de structuration individuée de l'expérience que de déstructuration des modes coutumiers de verbalisation et d'analyse du travail, en venant s'intercaler avant les réunions, moments légitimes de construction des représentations légitimes.

In this article, the two authors analyse an aid to writing about professional practice set up in an institution for problem children, during the working time. After a general first part where they attempt to describe the present stakes which bring the workers and their managements to look for the possibility of writing so as to formalise ans capitalise experience, they clarify the local conditions in which management and team of educators found a compromise. At last, they analyse the difficulties of writing : some proceed from the very process of putting into words the task of education of so called « autistics " young people. On the other hand, writing as a process causes the individual structuring of the experience as well as the destructuring of the conventional modes of wording ans analysis of the work, carried out during the usual meetings.

\section{INDEX}

Mots-clés : écriture, pratique, expérience, éducation spécialisée, éducateur, activité, direction, travail

Keywords : writing, practice, experience, specialized education, youth worker, activity, direction, work

\section{AUTEURS}

\section{VINCENT VALDELIĖVRE}

Vincent Valdelièvre, est éditeur de littérature d'expression populaire. Ancien formateur d'adultes, il anime des groupes d'écriture et participe à des formations de travailleurs sociaux, notamment pour la remise à l'écriture de salariés en formation continue. Titulaire d'un DEA, il est membre de l'équipe « communications organisationnelles » de GERICO/Lille3.

\section{PIERRE DELCAMBRE}

Pierre Delcambre, professeur en Sciences de l'Information et de la Communication à l'Université de Lille 3, anime les travaux de l'équipe « communications organisationnelles » de Gérico Il 
participe au GDR « Langage et travail » ainsi qu'au groupe de recherche sur les communications organisationnelles de la Société Française des Sciences de l'Information et de la Communication. Il travaille depuis une quinzaine d'années sur le terrain des établissements d'action sanitaire et sociale. Publication encours (sept 1997) Ecriture et communications de travail (Presses Universitaires du Septentrion). 